\title{
Amodiaquine induced agranulocytosis: inhibition of colony growth in bone marrow by antimalarial agents
}

\author{
ELIZABETH G H RHODES, JENNIFER BALL, IAN M FRANKLIN
}

\begin{abstract}
Bone marrow was cultured in vitro for colonies of granulocytes and macrophages five months after a patient had recovered from amodiaquine induced agranulocytosis. The addition of amodiaquine, chloroquine, and sulfadoxine to the culture was followed by a dose dependent inhibition of colony growth in the patient's marrow but not in normal control bone marrow. Colony growth was, however, unaffected by proguanil, pyrimethamine, and quinine.

These findings show that in vitro marrow culture may have important predictive value in some cases of drug induced agranulocytosis.
\end{abstract}

\section{Introduction}

Amodiaquine has been associated with agranulocytosis in a few patients taking both therapeutic and prophylactic doses of the drug.' A patient with amodiaquine related agranulocytosis was investigated five months after recovery to determine the in vitro sensitivity of colony formation in her bone marrow to amodiaquine and five other antimalarial agents.

\section{Patient and methods}

A 21 year old woman visited India for three months and took amodiaquine $200 \mathrm{mg}$ once weekly and proguanil $100 \mathrm{mg}$ daily as antimalarial prophylaxis. Three days before her return to England she became unwell and was admitted to hospital. She took her last dose of amodiaquine on that day but continued proguanil for a further month. A blood test showed a complete absence of neutrophils as the only abnormality. A bone marrow aspirate was almost totally devoid of myeloid precursors. After five days neutrophils reappeared in the peripheral blood, and by three weeks the white cell count was normal.

\footnotetext{
Department of Haematology, Queen Elizabeth Hospital, Birmingham B15 2TH

ELIZABETH G H RHODES, MRCP, senior registrar

JENNIFER BALL, medical laboratory scientific officer

IAN M FRANKLIN, PHD, MRCPATH, consultant haematologist

Correspondence to: Dr Franklin.
}

Marrow culture - Five months after the agranulocytosis bone marrow was aspirated for in vitro culture of granulocyte and macrophage colonies in agar by the method of Pike and Robinson, ${ }^{2}$ with and without the addition of six antimalarial agents. A normal control marrow sample was cultured in parallel. Feeder layers were prepared from peripheral blood leucocytes from the patient and two healthy controls. Amodiaquine, chloroquine, proguanil, pyrimethamine, sulfadoxine, and quinine were each added in three different concentrations to bone marrow cells and incubated for one hour at $37^{\circ} \mathrm{C}$ before plating in duplicate. The drug concentrations were based on therapeutic plasma concentrations, the middle one approximating to peak blood values achieved in normal subjects after a single standard therapeutic dose (for example, for amodiaquine $200 \mathrm{mg}$ ). After 14 days cultures were scored by counting the numbers of colonies containing more than 40 cells. The normal range for our laboratory is $50-200$ colonies $/ 2 \times 10^{5}$ cells plated.

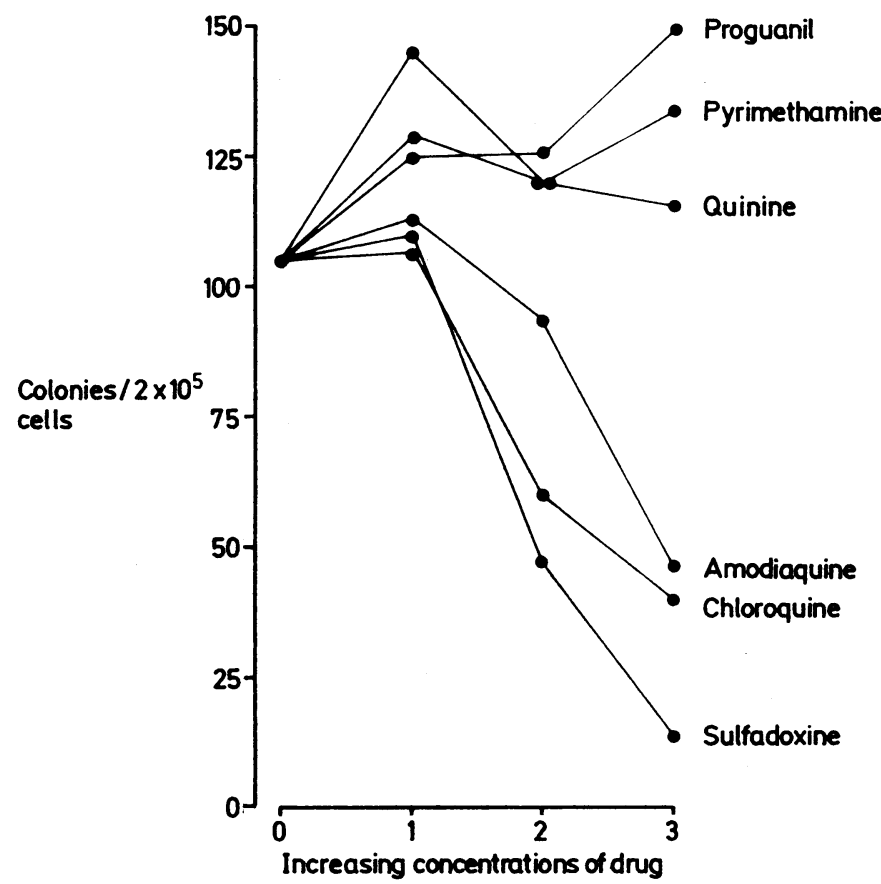

Colony scores at 14 days from patient's bone marrow cultured in presence of increasing concentrations of antimalarial agents. Concentrations 1,2 , and 3 represent $0.005,0.05$, and $0.5 \mathrm{mg}$ proguanil, amodiaquine, and chloroquine $/ \mathrm{l} ; 0.05,0.1$, and $0.5 \mathrm{mg}$ pyrimethamine $/ \mathrm{l} ; 1 \cdot 0,5 \cdot 0$, and $10.0 \mathrm{mg}$ quinine $/ 1 ; 50,200$, and $500 \mathrm{mg}$ of sulfadoxine/l. 


\section{Results}

The patient's and control bone marrow samples grew normally in the absence of antimalarial drugs. No antimalarial had any effect on normal marrow growth. Proguanil, pyrimethamine, and quinine had no effect on growth of the patient's marrow. Amodiaquine, chloroquine (a related 4aminoquinoline), and sulfadoxine, however, produced a dose dependent reduction in the number of colonies (figure).

\section{Discussion}

These results suggest that amodiaquine was responsible for the agranulocytosis since growth of colonies of granulocytes and macrophages was sensitive to this agent and recovery ensued on stopping the amodiaquine alone. Another patient with agranulocytosis had shown in vitro inhibition of growth of granulocytes and macrophages by amodiaquine early in the recovery phase.' A similar in vitro inhibitory effect was seen in a case of agranulocytosis induced by quinine. ${ }^{3} \mathrm{~A}$ specific effect on marrow colony growth is suggested, separate from any systemic abnormality of drug handling.

Our patient wished to know if she could take any medication to enable her to travel safely to areas where malaria was endemic. Clearly, $\underline{T}$ amodiaquine, chloroquine, and sulfadoxine were to be avoided. Though we could not be certain that the other drugs tested would be $\mathbb{\infty}$ safe, our results suggested that in vitro marrow culture may have important predictive value in some cases of drug induced agranulo- $\widehat{\widehat{O}}$ cytosis.

We thank Dr J B Wood, of Hereford County Hospital, for referring the patient and for permission to report the case. We also thank Imperial Chemical Industries PLC for supplying proguanil hydrochloride and chloroquine $\varrho$

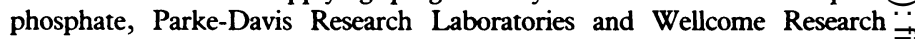
Laboratories for supplying amodiaquine hydrochloride, and Roche Products $\underset{\vec{\rho}}{\overrightarrow{2}}$ Ltd for supplying pyrimethamine and sulfadoxine, all in pure drug form.

\section{References} , Vincent PC. Amodiaquine-induced agranulocytosis: toxic effect of amodiaquine in bone marrow cultures in vitro. Br Med f 1973;i:458-60.

2 Pike BL, Robinson WA. Human bone marrow colony growth in agar-gel. $f$ Cell Physiol 1970;76:77-84. $\vec{\circ}$ 3 Sutherland R, Vincent PC, Raik E, Burgess K. Quinine-induced agranulocytosis: toxic effect of quinine bisulphate on bone marrow cultures in vitro. $\mathrm{Br}$ Med $\mathcal{f} 1977$;i:605-7.

(Accepted 4 February 1986)

\section{An early marker of fetal infection after primary cytomegalovirus infection in pregnancy}

\section{HAROLD STERN, GILLIAN HANNINGTON, JAMES BOOTH, DEBORAH MONCRIEFF}

\begin{abstract}
Fourteen patients with primary cytomegalovirus infection diagnosed by serological screening at antenatal attendances were examined for their responses in the lymphocyte transformation test against cytomegalovirus. Tests were done during pregnancy, shortly after the diagnosis of primary infection.

Eight women showed positive lymphocyte transformation responses and gave birth to uninfected babies. Six showed negative responses and four of the babies were born congenitally infected. Cellular immunity therefore plays a part in preventing intrauterine transmission of cytomegalovirus, and its depression after primary infection in the mother during pregnancy may be used as an early marker of fetal infection.
\end{abstract}

\section{Introduction}

Cytomegalovirus is the most common known cause of congenital infection in man and is as important, or more important, than rubella as a cause of handicap. In the United Kingdom $40-50 \%$ of women of childbearing age are seronegative and rather fewer than $1 \%$ of these undergo primary infection at some time during pregnancy.$^{1.3}$ Following primary infection transmission of virus to

\footnotetext{
Departments of Virology and Obstetrics, St George's Hospital Medical School, London SW17 0RE

HAROLD STERN, FRCPATH, professor of virology

GILLIAN HANNINGTON, PHD, research fellow in virology

JAMES BOOTH, PHD, MRCPATH, senior lecturer in virology

DEBORAH MONCRIEFF, MRCOG, senior registrar in obstetrics

Correspondence to: Professor Stern
}

the fetus occurs in $25-50 \%$ of cases. ${ }^{14}$ Only $10-14 \%$ of the infected ${ }_{\infty}^{\infty}$ fetuses, however, suffer important sequelae, usually neurological damage or sensorineural deafness. ${ }^{5.7}$ The overall risk of a baby being born with congenital abnormality after primary infection in the mother is therefore relatively low, probably $5 \%$ or less. Hence it has been advocated that, as there is no means of identifying the $\frac{\circ}{\Phi}$ pregnancies most at risk of resulting in a handicapped baby, neither $\varrho$ screening for primary infection nor termination, as offered for $\overrightarrow{\overrightarrow{0}}$ rubella, is justified.$^{89}$

Women who have given birth to babies congenitally infected with cytomegalovirus have been found to have depressed cell mediated immunity to cytomegalovirus, demonstrable by the lymphocyte transformation test, ${ }^{10}$ with a tendency to greater depression in the mothers who had given birth to infants with abnormalities. ${ }^{12}$ All $;$ these studies, however, were carried out only after delivery, usually many months later. This study was undertaken to see whether $\delta$ measurement of lymphocyte transformation response during pregnancy, at the time of occurrence of the primary infection, could 9 predict the likelihood of intrauterine infection. Such information would be invaluable in clinical management.

\section{Patients and methods}

Since July 1982 women attending the antenatal booking clinics of St $\bar{O}$ George's Hospital and the South London Hospital have been routinely 0 screened for serum cytomegalovirus specific IgG by indirect enzyme linked $\stackrel{D}{\Phi}$ immunosorbent assay. ${ }^{13}$ Positive serum samples were re-examined for $\stackrel{\mathbb{Q}}{+}$ cytomegalovirus specific IgM, also by indirect enzyme linked immuno- 0 sorbent assay, to determine whether infection had occurred within the preceding two to three months (see below). Most of the women were first $\overrightarrow{\mathbb{D}}$ seen at about 12 weeks' gestation. Women who were seronegative were re- $\frac{?}{\mathbb{P}}$ examined at intervals during the pregnancy for seroconversion. When $\stackrel{\mathbb{Q}}{\varrho}$ primary infection was diagnosed, whether by seroconversion or by the presence of cytomegalovirus specific $\operatorname{IgM}$, the patient was recalled as soon as $\varnothing$ possible and a lymphocyte transformation test carried out; this was repeated $\frac{0}{0}$ at further intervals during pregnancy and again at term. All women enrolled in the investigation were made fully aware of its nature, and follow up examinations were undertaken only with their consent; about a third of the 